\title{
Multispectral lensless digital holographic microscope: imaging MCF-7 and MDA-MB-231 cancer cell cultures
}

\author{
James P. Ryle ${ }^{\mathrm{a}, \mathrm{b}, \mathrm{c}}$, Karen M. Molony ${ }^{\mathrm{d}}$, Susan McDonnell ${ }^{\mathrm{e}}$, Thomas J. Naughton ${ }^{\mathrm{d}, \mathrm{f}}$ \\ and John T. Sheridan ${ }^{\mathrm{a}, \mathrm{b}, \mathrm{c},{ }^{*}}$ \\ ${ }^{\mathrm{a} U C D}$ Communications and Optoelectronics Research Centre \\ ${ }^{\mathrm{b}}$ SFI Strategic Research Centre in Solar Energy Conversion, \\ ${ }^{\mathrm{c}}$ School of Electronic, Electrical and Mechanical Engineering, \\ College of Engineering, Mathematical and Physical Sciences, \\ University College Dublin, Belfield, Dublin 4, Ireland. \\ ${ }^{\mathrm{d}}$ Department of Computer Science, National University of Ireland Maynooth, \\ Maynooth, County Kildare, Ireland. \\ e School of Chemical and Bioprocess Engineering, \\ College of Engineering, Mathematical and Physical Sciences, \\ University College Dublin, Belfield, Dublin 4, Ireland. \\ ${ }^{\mathrm{f}}$ University of Oulu, RFMedia Laboratory, Oulu Southern Institute, \\ Vierimaantie 5, 84100 Ylivieska, Finland.
}

\begin{abstract}
Digital holography is the process where an object's phase and amplitude information is retrieved from intensity images obtained using a digital camera (e.g. CCD or CMOS sensor). In-line digital holographic techniques offer full use of the recording device's sampling bandwidth, unlike off-axis holography where object information is not modulated onto carrier fringes. Reconstructed images are obscured by the linear superposition of the unwanted, out of focus, twin images. In addition to this, speckle noise degrades overall quality of the reconstructed images. The speckle effect is a phenomenon of laser sources used in digital holographic systems. Minimizing the effects due to speckle noise, removal of the twin image and using the full sampling bandwidth of the capture device aids overall reconstructed image quality. Such improvements applied to digital holography can benefit applications such as holographic microscopy where the reconstructed images are obscured with twin image information. Overcoming such problems allows greater flexibility in current image processing techniques, which can be applied to segmenting biological cells (e.g. MCF-7 and MDA-MB231) to determine their overall cell density and viability. This could potentially be used to distinguish between apoptotic and necrotic cells in large scale mammalian cell processes, currently the system of choice, within the biopharmaceutical industry.
\end{abstract}

Keywords: In-line digital holography, lensless microscope, Gabor microscopy, wavefront reconstruction

*Corresponding author: john.sheridan@ucd.ie , phone: +353-1-716-1927

Optics and Photonics for Information Processing III, edited by Khan M. Iftekharuddin, Abdul Ahad Sami Awwal, Proc. of SPIE Vol. 7442, 744206 · C 2009 SPIE · CCC code: 0277-786X/09/\$18 · doi: 10.1117/12.826882 


\section{INTRODUCTION}

The first lensless holographic system was 'serendipitously' discovered by Gabor in the late 1940's to overcome lens aberrations in electron microscopy [1]. Since its inception the technique has been applied to electron holography [2], [3] as well as microscopy in the study of 3D trajectories of plankton (copepod nautilplius) [4], imaging microspheres [5], [6], [7] and for the imaging of cancer cells[7],[8] as well as tracking particles in space and time[9] and inspection of deposits on silicon wafers[10]. The technique has been employed to image objects using sources of varying wavelengths and degrees of coherence [6], [7], [10], [11], [12], [13]. The speckle effect is a phenomenon associated with coherent sources. A speckled pattern, due to the mutual interference of wavefronts can arise when light is reflected from rough surface [14]. Using optical sources with short coherence lengths such as Light Emitting Diodes (LED's), the effects of speckle decreases. Owing to low coherence, lower visibility interference fringes occur with the resulting decreased fringe visibility yielding lowered resolving power of the Digital In-line Holographic Microscope (DIHM) [6].

In a lensless setup, the scattered light from the sample is the object beam, while unscattered incident light acts as the reference beam. The superposition of these two beams results in an interference pattern is recorded by a digital camera such as a Charged Coupled Device (CCD) or Complementary Metal Oxide Semiconductor (CMOS) sensor. From this two-dimensional intensity image, the object wavefront is numerically reconstructed to give a full hologram as a complex image with both amplitude and phase information. Using multiple computer-based amplitude reconstructions at differing depths, a three-dimensional (3D) surface of imaged features is rendered[15]. Greater lateral and longitudinal resolution is achievable by increasing the systems numerical aperture (NA) [16].

In digital holographic systems, the twin image is present and obstructs part of the reconstructed infocus real image. Methods have been devised that minimise the effects of the twin image in in-line holographic systems [3], [17]. By capturing a number of interference patterns with varying phase shifts of the reference beam, the DC, and twin image terms can be eliminated [17]. A variation of this method exists where the reference beam phase is altered with recordings of the holograms being made at different planes [18], [19]. In respect of the lensless system described in this paper, however, the twin image is considered to be smeared out over space. Thus, similar to Fraunhofer holography, the effects of the twin image are assumed to be negligible [16]. In off-axis geometries, the twin image is separated from the real image by shifting the information in space [20], [21]. We note that sampling the fringe pattern containing the spatially modulated real and twin images as well as the DC component decreases the overall resolution of the holographic system [22]

High magnification microscopes have limited depths of focus. Using a single hologram, it is possible to numerically focus a reconstructed image at multiple depths [16]. Biological samples are quasi-transparent and often need to be stained to aid in microscopic imaging, or by using-using phase contrast methods, the unstained quasi-transparent sample is successfully imaged. Eliminating the need to manually focus conventional microscope systems combined with access to both sample amplitude and phase information offers the potential of greater flexibility when processing biological samples images.

Cells lines such as Chinese Hamster Ovary (CHO), Baby Hamster Kidney (BHK) and mouse myeloma (NS0) cells are grown in large scale $(>12,500$ litre bioreactors) in the biopharma industry for the production of biopharmaceuticals like Embrel (Wyeth) used as an anti-arthritis treatment, Herceptin (Genentec) used in breast cancer treatment or Epogen (Amgen) used to increase and maintain a high level of red blood cells. Cell cultures are grown in a growth medium containing nutrients. They are either anchorage dependant or independent. Anchorage independent cells grow in suspension and are constantly agitated and mixed throughout the growth vessel. Anchorage dependant cells grow attached to a substrate. These kind of cells are known as adherent cells and include the MCF-7 [23] and MDA-MB-231 [24] cancer cell lines described in this paper.

Cell numbers can grow exponentially provided the environment is suitable [25]. This involves provision of: 1) sufficient nutrients and Carbon Dioxide $\left(\mathrm{CO}_{2}\right) ; 2$ ) a constant temperature of $37^{\circ} \mathrm{C}$; and 3) space to multiply. Should any one of these growth parameters be compromised, the cells will not grow at an exponential rate and productivity will be reduced. If optimum growth conditions continue to deteriorate, cells will die either by unnatural death, i.e. necrosis or programmed cell death, i.e. apoptosis. In large scale mammalian cell processes, the ability to count the total number of cells and to distinguish between living (viable), dead (non-viable) cells and other bodies provides a cell viability 
estimation. Confluency refers to the density of cells in their growth environment. For adherent cells, 100\% confluency means the entire growth surface is occupied by cells. This will effectively limit the growth of further cells.

Until recently counting cells required a skilled technician and a haemocytometer to manually count cells. Using the area of a predefined grid pattern etched onto the bottom of the haemocytometer and given a known volume of the culture sample as well as an average count of cells, an estimate of cell density can be determined. Staining the cells with a chemical (e.g. Trypan Blue), an estimate of the number of unstained viable cells can be made. Trypan Blue leaks into dead cells membrane and stains the nonviable cells. Recently, an automated system, Vi-Cell (Beckman Coulter) was developed to perform cell counts using Trypan Blue assay analysis with two-dimensional hyper-spectral incoherent image analysis. The ability to count cell numbers and estimate cell densities enables researchers to determine the optimum time to subculture cells into larger volume growth environments.

In this paper, a compact Digital In-line Holographic Microscope (DIHM), based on the method first proposed by Gabor in the late 1940's using coherent visible light, is described. A Helium-Neon (HeNe) laser (Research Electro-Optics Inc.) was used as the light source. One of its attributes is that it produces multiple illuminating wavelengths making multispectral imaging possible. We capture and process holograms of adherent MCF-7 and MDA-MB-231 cancer cells extracting both amplitude and phase images. From the reconstructed images it is possible to localise areas of interest and segment cells to provide a cell count as well as percentage density in an effort to estimate the level of confluency of the samples.

\section{PRINCIPLE OF OPERATION}

The primary lightsource from a laser emitting light at wavelength ' $\lambda$ ' is focused onto a pinhole by a microscopic objective lens. The pinhole acts to spatially filter the light, removing higher order aberrations and phase front distortions, thus providing the secondary illuminating lightsource. This point source emits a spherical wavefront. A sample is placed at a distance ' $z$ ' away from the pinhole, with the digital recording device (e.g. CCD or CMOS camera) immediately behind the sample, a distance ' $L$ ' away from the pinhole (Fig. 1) such that $L>z$. The size of the image formed at the camera plane is dependant on the geometrically magnified diffraction pattern of the spherical wavefront; hence the system is considered a lensless imaging system [REP04]. The magnification of the system is given by the ratio of $L / z$, while the effective reconstruction distance used is given by,

$$
D_{\text {eff }}=\frac{L}{z}(L-z) \text {. }
$$

Unscattered light from the sample acts as the reference beam $A_{\text {ref }}(x, y, 0)$, while light scattered by the object is the object beam, $A_{\mathrm{obj}}(x, y, 0)$. The superposition of the resulting diffraction patterns $H(x, y, 0)$ resulting from the interference of these two beams is recorded by the camera at $z=0$,

$$
H(x, y, 0)=\left|A_{\text {ref }}(x, y, 0)+A_{o b j}(x, y, 0)\right|^{2} .
$$

where $x$ and $y$ are spatial coordinates.

Reconstructing this hologram results in a 'brightfield' amplitude image with constant DC background illumination present. In an effort to remove this DC term, i.e. to numerically correct for the intensity variations of the secondary source, a normalised contrast hologram [2], [3], [5], [12], $H_{\mathrm{c}}(x, y, 0)$, is derived by first subtracting an unscattered reference illumination $I_{0}(x, y, 0)$ from the hologram and then dividing by the square root of this unscattered reference illumination secondary source,

$$
H_{c}(x, y, 0)=\frac{H(x, y, 0)-I_{0}(x, y, 0)}{\sqrt{I_{0}(x, y, 0)}} .
$$


Thereafter, the captured hologram is numerically reconstructed using the Kirchhoff-Helmholtz algorithm[2], [6], [12]. From this two-dimensional hologram, based on the image recorded by the camera, it is possible to obtain both amplitude and phase information at one plane,

$$
K(x, y, L-z)=\iint H(x, y, 0) P\left(x, y, D_{e f f}\right) d x d y,
$$

with the diffraction in free space $P\left(x, y, D_{e f f}\right)$ given by,

$$
P\left(x, y, D_{\text {eff }}\right)=\frac{i \pi}{\lambda\left[\left(x_{0}-x\right)^{2}+\left(y_{0}-y\right)^{2}+D_{\text {eff }}{ }^{2}\right]} \exp \left\{\frac{i 2 \pi}{\lambda}\left[\left(x_{0}-x\right)^{2}+\left(y_{0}-y\right)^{2}+D_{\text {eff }}^{2}\right]\right\},
$$

where $x_{0}, y_{0}$ are the central coordinate points and $D_{\text {eff }}$ is the effective reconstruction distance.

The numerically reconstructed wavefront $K(x, y, L-z)$ is a complex image of the captured hologram $H(x, y, 0)$ with the amplitude given by the results in complex wavefront containing both amplitude and phase information.

The amplitude and phase information are obtained by from the absolute and arctangent of the numerically reconstructed complex image respectively. The ensuing phase map from the phase information contains discontinuities where the sample height is greater than the wavelength. Therefore this $2 \pi$ wrapped image, where $2 \pi$ refers to one cycle of the optical wavelength requires phase unwrapping. Phase unwrapping is the process of adding or subtracting integer multiples of $2 \pi$ to the discontinuous phase map removing the discontinuities [26], [27]. Using two phase images captured using different illuminating wavelengths, it is possible to construct a phase image as if it were obtained using a much greater wavelength [28]. This is achieved by comparing the discontinuities in phase images where the only altered variable is the illuminating wavelength. The resulting phase image has fewer phase discontinuities in that it appears as if it was obtained using an illumination source of a much greater wavelength. For example, if two wavelengths, $633 \mathrm{~nm}$ and $543 \mathrm{~nm}$, were used and the resulting phase images compared using this method. It would appear as though the new phase image was obtained using a $3.81 \mu \mathrm{m}$ souece. This is known as the synthetic or equivalent wavelength ' $\Lambda$ ',

$$
\Lambda=\frac{\lambda_{1} \lambda_{2}}{\left|\lambda_{1}-\lambda_{2}\right|},
$$

where $\lambda_{1}$ and $\lambda_{2}$ are the differing illuminating wavelengths.

In the lensless setup, the Numerical Aperture (NA) of the system is defined as $N A=\eta \sin (\theta)$, where ' $\eta$ ' is the refractive index of the propagating medium and $\theta$ is the half angle of illumination. Considering the geometry of the setup, the relationship for the NA is obtained using the limiting factor of the camera chip width and distance of propagation from the point source to the camera. Assuming a refractive index of 1 and very small angles of illumination (paraxial conditions) such that $\sin (\theta) \approx \theta$, the relationship for NA becomes,

$$
N A=\frac{W}{2}\left[\left(\frac{W}{2}\right)^{2}+(L)^{2}\right]^{-\frac{1}{2}},
$$

where $W$ is the width of the camera chip and ' $L$ ' is the distance from the point source to the camera face.

Increased NA has several benefits to the system resolution, most noticeably an increase in both lateral and longitudinal resolutions [16]. Lateral resolution ' $\delta x$ ' refers to the ability to image two separate points inplane with the camera while longitudinal or depth resolution ' $\delta z$ ' refers to the ability to distinguish between two separate points in separate planes. Thus lateral and longitudinal resolution is defined as,

$$
\delta x \geq \frac{\lambda}{2 N A} \quad \text { and } \quad \delta z \geq \frac{\lambda}{2(N A)^{2}},
$$


where $\lambda$ is the illuminating wavelength and NA is the numerical aperture of the DIHM.

Increased lateral and longitudinal resolution is obtained by using a smaller illuminating wavelength, or by increasing the NA. Increasing the NA, as defined in Eqn. 7 is achieved by increasing the variable ' $W$ ' corresponding to the width of the camera, or decreasing the propagation distance from the point source to the camera.

\section{METHODOLOGY}

A schematic diagram of the setup used in these experiments is shown in Fig. 1. In our system, a tuneable Helium-Neon (HeNe) laser (Research Electro-Optics Inc.) is used as the primary light source. This can be tuned separately to emit wavelengths of $633 \mathrm{~nm}, 612 \mathrm{~nm}, 604 \mathrm{~nm}, 594 \mathrm{~nm}$ and $543 \mathrm{~nm}$. Light from the laser is focused by a 20X microscopic objective lens onto a $2 \mu \mathrm{m}$ pinhole. The emitted secondary source is scattered by the sample and captured using a digital camera [Imperx 1M48 (1000 x 1000 pixels)].

The monochromatic digital camera with a pixel pitch of $7.4 \mu \mathrm{m}$ to capture holograms. Numerical reconstruction was performed on a laptop with an Intel P8400 Core Duo processor of clock speeds of $2.26 \mathrm{GHz}$ per processer and with 3 GB of RAM. The MatLab 7.4.0 (R2007a) (Mathworks Inc.) programming environment was used to implement the Kirchhoff-Helmholtz reconstruction algorithm.

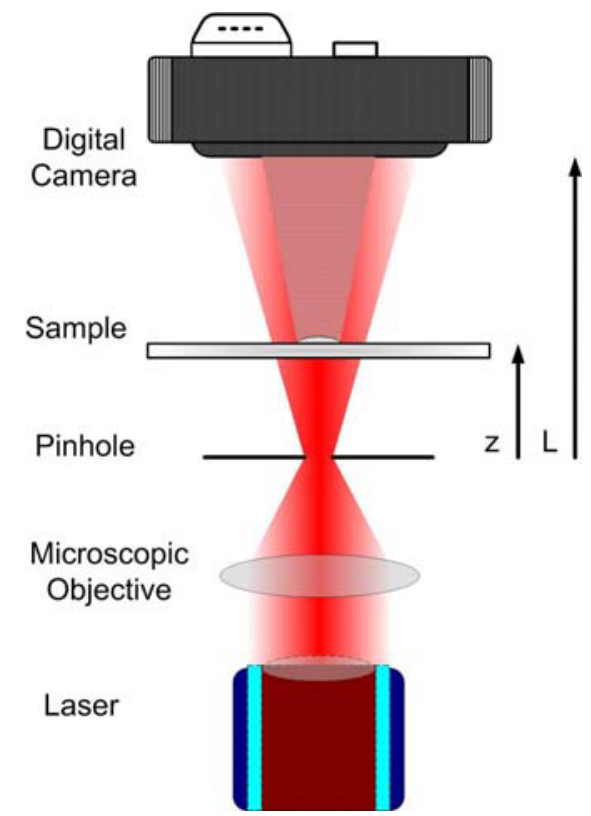

Figure 1: System schematic for a Digital In-line Holographic Microscope.

For this paper, the mammalian breast cancer MCF-7 and MDA-MB-231 cell lines were routinely grown in Dulbecco's Modified Eagle's Medium (DMEM) supplemented with 10\% foetal calf serum. Cells were cultured in T25 flasks at $37^{\circ}$ $\mathrm{C}$, with a $5 \% \mathrm{CO}_{2}$ atmosphere. Between $0.5 \mathrm{ml}$ and $1 \mathrm{ml}$ of the $\mathrm{T} 25$ flask containing each of the cell lines was subcultured into each well of a Lab-Tek II 4-well sterile chambered glass slide. Once the cells had adhered to the glass slide, they were chemically fixed and the chamber walls were removed. This yielded a total of four wells, two wells of each cell line. To aid imaging of the cell lines on a conventional compound microscope, one well of each of the quasitransparent samples were stained using $0.25 \%$ crystal violet. This should not be confused with Trypan blue which stains non-viable cells and is used to estimate cell viability. 


\section{RESULTS}

We now present the processed experimental results obtained using the system described in the previous section. For illustration purposes the following results were obtained using our HeNe laser emitting at $633 \mathrm{~nm}$. Multispectral results are presented later in this section.

\subsection{MCF-7 reconstruction}

Before inserting the glass slide containing the cell samples into the system, an image of the reference illuminating secondary wavefront was captured. After this, holograms were captured and stored on the computer for later offline processing. Constructing the contrast hologram, as much of the unwanted DC term as possible was removed. Fig. 1(a) shows this capture as well as the resulting contrast hologram in Fig. 2(b) with the sample placed a distance $z=2.5 \mathrm{~mm}$ away from the secondary source of the pinhole. The camera was located a distance $L=35 \mathrm{~mm}$ from the secondary source. Given the ensuing magnification of 14, the numerically reconstructed amplitude, Fig. 2(c) and phase, Fig. 2(d) are also shown.

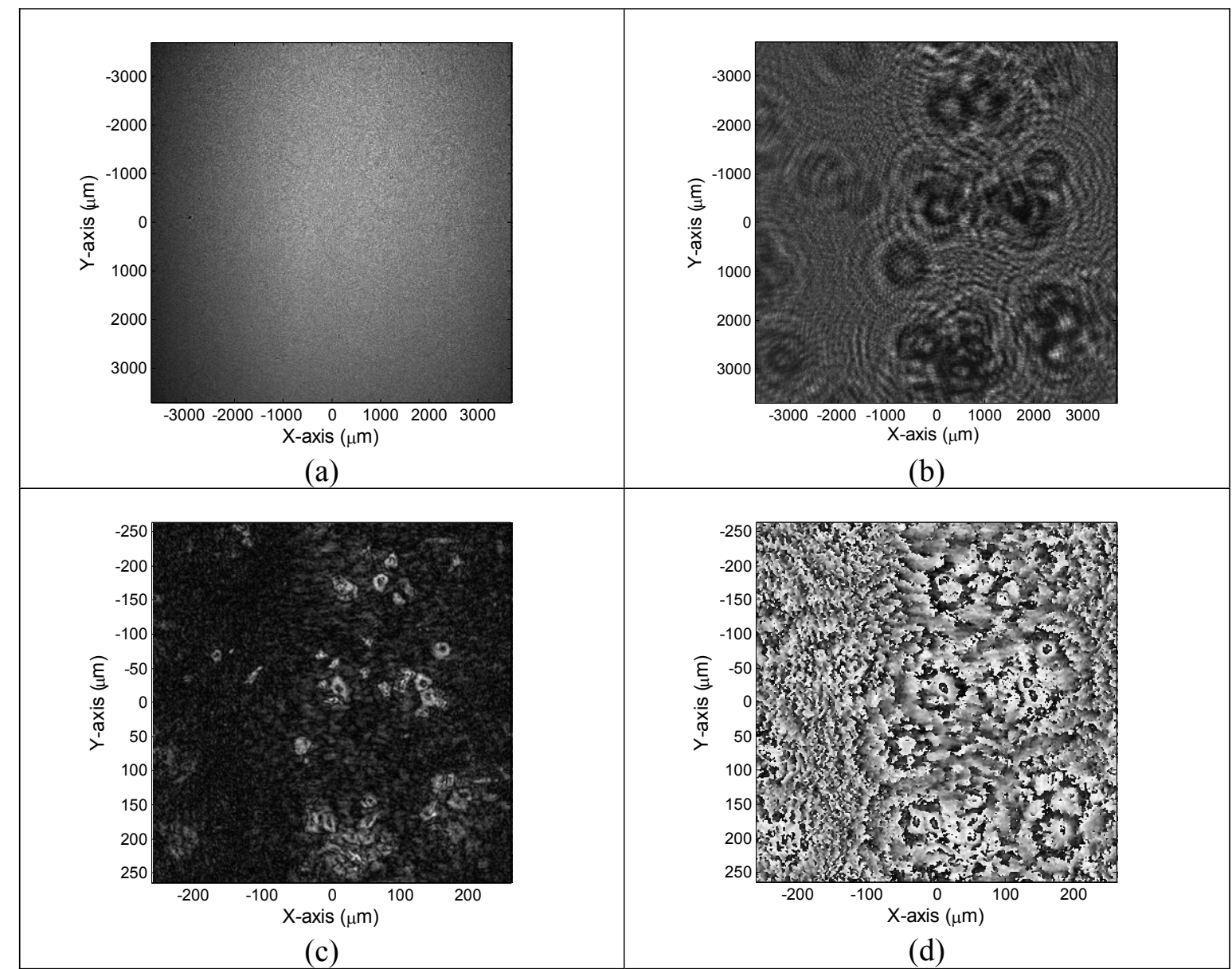

Figure 2: MCF-7 cell culture, (a) reference illumination without any sample (b) contrast hologram, (c) darkfield amplitude and (d) wrapped phase, $z=2.5 \mathrm{~mm}, L=35 \mathrm{~mm}$, Magnification $=14$, NA $=0.1055$.

In the amplitude image, Fig. 2(c) the in focus objects appears bright with a dark non-uniform background. Areas of interest in the phase image, Fig. 2(d) are present, but the wrapped phase hinders straightforward identification. Applying a two dimensional (2D) phase unwrapping algorithm [27] to the data in Fig. 2(d) results in a poor representation of the 
objects' true phase. As can be seen in the phase image, phase discontinuities are present throughout the entire image. Applying the above phase unwrapping algorithm to our phase image yielded an image with indistinguishable objects.

\subsection{MDA-MB-231 reconstruction}

A similar approach was adopted to image the MDA-MB-231 sample as described in the Section 4.1. However, unlike the previous example, we generated the reference image numerically by performing a lowpass filtering operation on the original hologram. By simulating the reference illumination intensity, the process of physically capturing two images per reconstruction is eliminated. First, the 2D Fast Fourier Transform (FFT) algorithm was applied to the $1000 \times 1000$ pixel hologram, after which a lowpass $3 \times 3$ pixel mask was applied to DC component of the image Performing the inverse 2D FFT algorithm on the DC passed image resulted in the simulated reference illumination intensity, as shown in Fig. 3(a). The resultant contrast hologram is shown in Fig. 3(b). In this instance, the sample was located at $z=22 \mathrm{~mm}$ from the secondary source and the camera was positioned a distance $L=125 \mathrm{~mm}$ from the secondary source. The magnification in this example was lowered to 5.68 as a result of altering the distances $L$ and $z$ as per Eqn. 1.

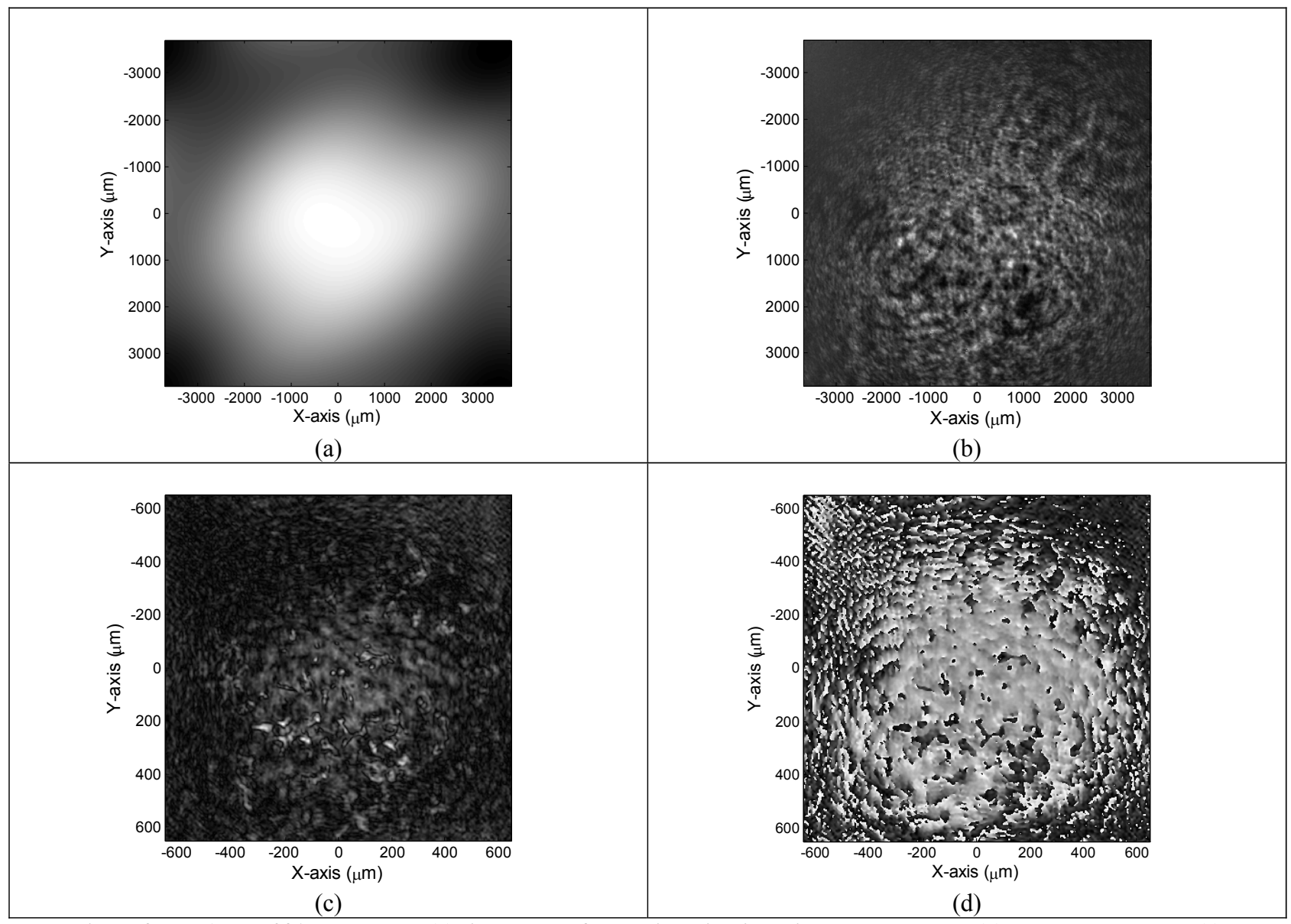

Figure 3: MDA-MB-231 cell culture, (a) simulated reference illumination without any sample (b) contrast hologram, (c) darkfield amplitude and (d) wrapped phase, $z=22 \mathrm{~mm}, L=125 \mathrm{~mm}$, Magnification $=5.68$ and $\mathrm{NA}=0.031$.

The amplitude image contains bright MDA-MD-231 cells. However, the DC component in the background appears to be present. This suggests the simulated reference illumination obtained by lowpass filtering the hologram is not an exact match for the capture reference illumination as shown in the previous results for MCF-7. The image representing the object phase in Fig. 3(d) contains fewer phase discontinuities than the previous numerically reconstructed phase image for MCF-7 cells, Fig. 2(d). Accordingly cell locations are more readily distinguishable by the human eye. 


\section{3 Multispectral imaging}

Capturing holograms at different wavelengths using a monochromatic camera requires multiple sequential captures. The reference illumination intensity is typically altered through changes in the wavelength. In order to obtain the reference illumination using our simple system, the sample must be removed before each capture and inserted at the exact location it was prior to its removal. In such a sequential multispectral imaging system, replacing the slide precisely in its previous position cannot be achieved. Hence, we employed the technique introduced in Section 4.2 of simulating the reference illumination from a lowpass filtered image of the captured hologram. Assuming our simulated reference illumination intensity is a good representation of the true reference illumination intensity, sequential multispectral imaging is possible using a monochromatic camera.

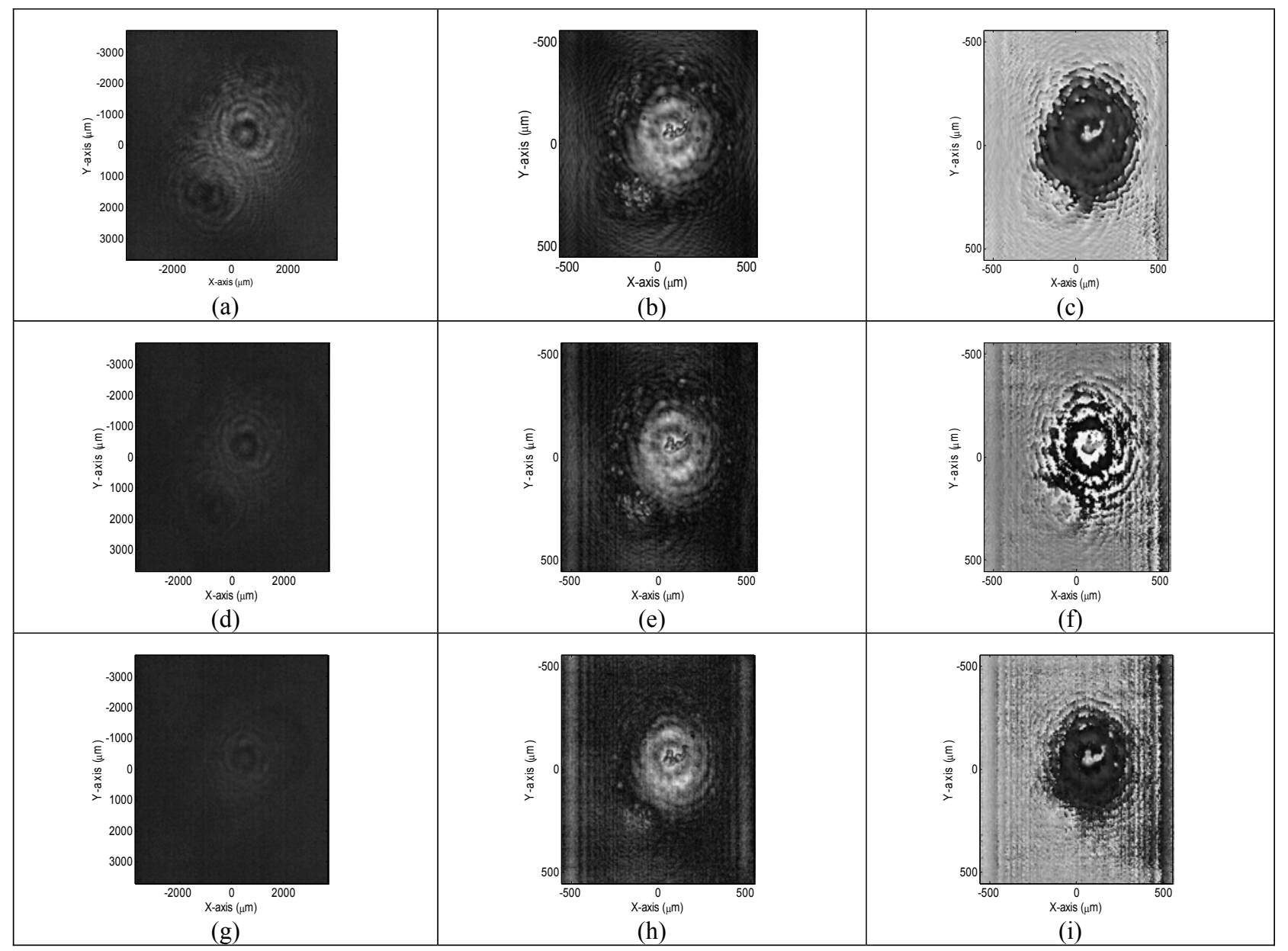

Figure 4: Multiple images of the same region using three different illuminating wavelengths, (a)-(c) at 633nm, (d)-(f) at 604nm and (g)-(i) at $543 \mathrm{~nm}$. Holograms (a), (d) and (f) of MCF-7 cells with reconstructed amplitude (b), (e) and (h) and phase information (c), (f) and (i). Experimental parameters were $z=12 \mathrm{~mm}, L=80 \mathrm{~mm}$, Magnification $=6.67$ and NA=0.049.

Contrast holograms, amplitude and phase images are shown in Fig. 4 using our tunable HeNe laser with wavelengths of $633 \mathrm{~nm}$, Figs. 4(a - c); $604 \mathrm{~nm}$, Figs. 4(d - f) and $543 \mathrm{~nm}$ Figs. 4(g - i). The rated power of the laser as per its datasheet was $4.0 \mathrm{~mW}, 0.5 \mathrm{~mW}$ and $0.3 \mathrm{~mW}$ respectively for $633 \mathrm{~nm}, 604 \mathrm{~nm}$ and $543 \mathrm{~nm}$ wavelengths The images in Fig. 4(ac) were obtained with the highest power beam. $\lambda=633 \mathrm{~nm}$ yields greater fringe visibility in the contrast hologram. Fig. 4(a) also provides better resolution in the reconstructed amplitude image, Fig. 4(b). Comparing the phase images, i.e., 
Fig. 4(c) obtained using $\lambda=633 \mathrm{~nm}$ at $4.0 \mathrm{~mW}$, and Fig. 4 (i) obtained using $\lambda=543 \mathrm{~nm}$ at $0.3 \mathrm{~mW}$, the latter appears to provide the roughest phase representation.

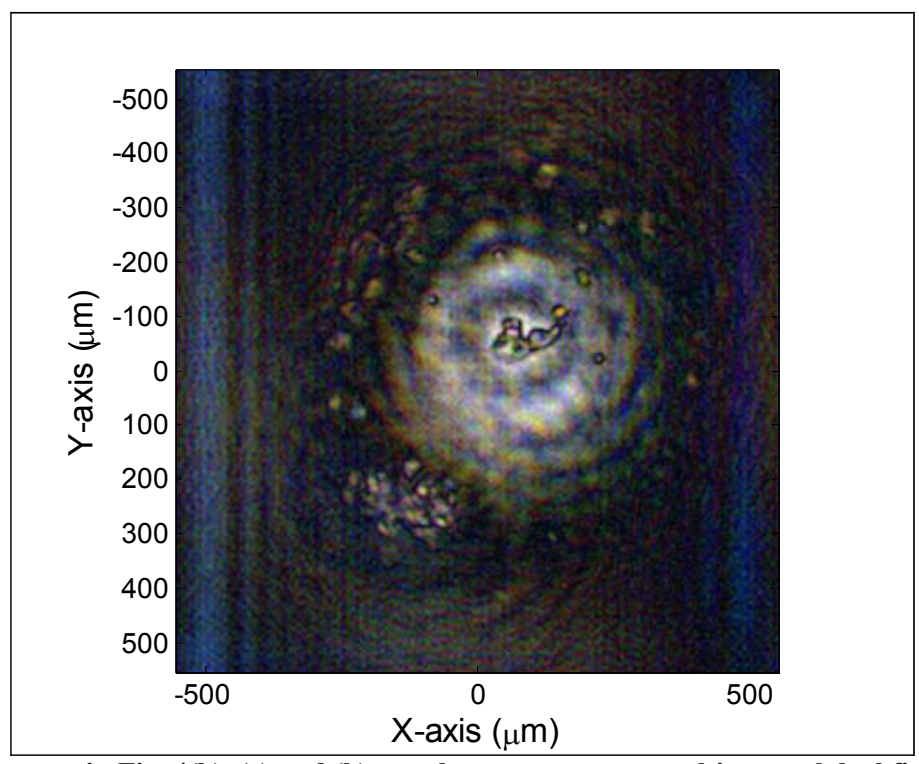

Figure 5: Combining the images in Fig. 4(b), (e) and (h) together to generate a multispectral darkfield image of MCF-7 cancer cells. Experimental parameters used were $z=12 \mathrm{~mm}, L=80 \mathrm{~mm}$, Magnification $=6.67$ and NA=0.049.

The recombined multispectral amplitude image is shown in Fig. 5. This is a result of combining the three amplitude images shown in Figs. 4(b), (e) and (h) corresponding to wavelengths of $633 \mathrm{~nm}, 604 \mathrm{~nm}$ and $543 \mathrm{~nm}$ respectively. Combining multispectral amplitude images, such as the image shown in Fig. 5, could assist cell viability estimation allowing detection of Trypan blue stained non-viable cells adding to the advantages of using a DIHM to monitor cell culturing processes.

\subsection{Image segmenting estimating the level of confluency}

In order to estimate the level of confluency in the MCF-7 sample, the density of cells is required. An adaptive threshold algorithm [15] was used to remove unwanted out of focus features. These included background noise and out of focus features, as well contributions from speckle noise and the twin image. In the reconstructed contrast image, in focus features are bright with respect to the background. A simple method to segment the image is to apply a threshold to each pixel value. This determines infocus features and assigns them as foreground and vice-versa for the background. However, this method does not account for non-uniform illumination. Therefore an adaptive threshold was used to determine regions of interest, while a hierarchical threshold algorithm was used to remove features of inconsequential size using a windowed filter. In our example, these features are relatively smaller than the average cell size. To illustrate this, the amplitude image in of the fixed cells presented in Fig. 2(c) is used (Fig. 6). 


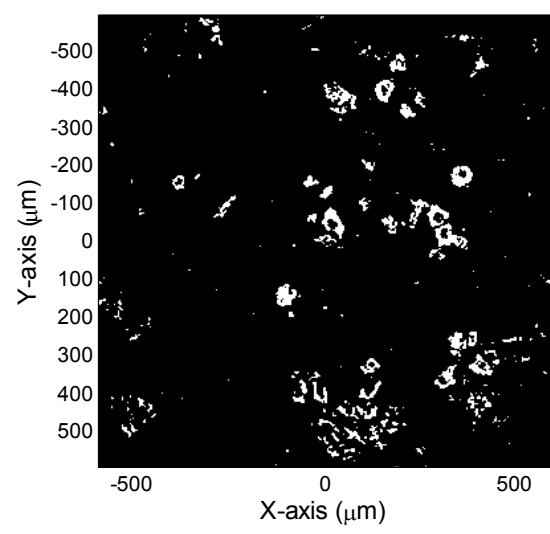

(a)

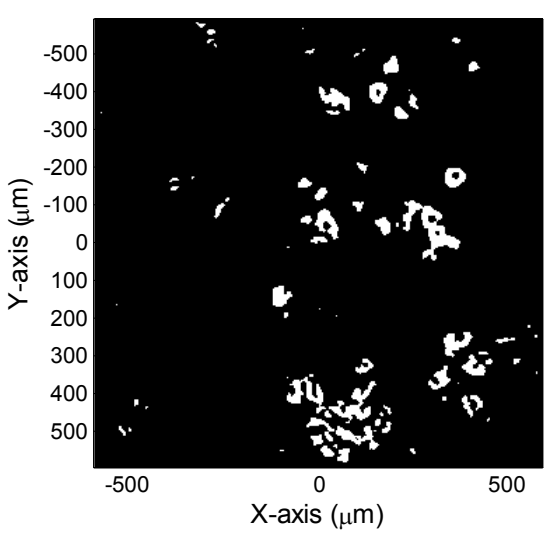

(b)

Figure 6: Segmented amplitude reconstruction image of MCF-7 after application of (a) the adaptive threshold algorithm and (b) the hierarchial threshold algorithm from the data shown in image Fig. 2(c).

An adaptive threshold algorithm with a threshold value of 200 was initially applied to an 8-bit image. The resulting binary image is shown in Fig. 6(a). Areas of interest appear white with respect to the dark background. The hierarchial threshold algorithm was then applied to the data in image of Fig. 6(a) using a summed combination of results using square windows of $3 \times 3,7 \times 7$ and $11 \times 11$ pixels. The ensuing image in Fig. 6(b) approximates the area of interest where cells are. Summing the total number of white pixels results in an estimate of $3.97 \%$ for the level of confluency of the MCF-7 sample presented here.

\section{DISCUSSION AND CONCLUSION}

A simple Digital In-line Holographic Microscope (DIHM) with multispectral imaging capabilities, based on the principle first proposed by Gabor in the late 1940's, was developed. Using this system, it is possible to capture holograms of the MCF-7 and MDA-MB-231 mammalian breast cancer cell lines. The DIHM is considered a lensless system and we have shwon magnification up to $14 \mathrm{X}$ based on holograms from a geometrically magnification diffraction patterns.

The first set of results presented in Fig. 2 were obtained using the conventional contrast hologram technique. This required two camera captures - the reference illumination intensity as well as the geometrically magnified diffraction pattern or hologram. Our second set of results in Fig. 3 only used one capture, with the reference illumination intensity simulated from a low passed filtered image of the hologram. This method enabled sequential multispectral imaging without removing the sample to record the reference illumination intensity for each wavelength used, as shown in Fig. 4 .. Combining the resulting multispectral images, a multispectral darkfield image of the cell was created. Such multispectral DIHM reconstructed amplitude images could be used for Trypan blue cell viability investigations. However, one failing of these preliminary results is that the data presented here does not take into account chromatic aberrations.

In this paper, we have reviewed some of the current techniques used to count cells and presented a simple image analysis technique using DIHM data. By performing adaptive thresholding, we have estimated the level of confluency of a MCF7 sample from a numerically reconstructed amplitude image of a captured hologram. With some processing of the phase information, i.e. successful phase unwrapping, this method could be extended to incorporate an estimate from the phase information as well from DIHM phase images. Multispectral DIHM allows the construction of phase images using a synthetic or equivalent wavelength. This could extend the continuous region before applying $2 \mathrm{D}$ phase unwrapping algorithms.

In large scale mammalian cell processes which have had regulatory approval and are currently the system of choice within the biopharmaceutical industry, there is a need to determine cell count or cell density as well as cell viability. It is 
important that cells be viable (alive) as these cell produce biopharmaceuticals. Thus there is a need to distinguish between dead or apoptotic cells, necrotic bodies and viable cells in determining a meaningful result from a DIHM system. By using both amplitude and phase information, it is hoped to extend our system to perform viable cell counts using DIHM.

\section{ACKNOWLEDGMENTS}

The authors acknowledge the support of Enterprise Ireland and Science Foundation Ireland. They also thank the Irish Research Council for Science, Engineering and Technology for their support under the national development plan. James Ryle wishes to thank SPIE for supporting conference attendance through the Student Chapter Officer Travel Grant programme. He also acknowledges financial support from UCD's Seed Funding Scheme.

\section{REFERENCES}

1. D. Gabor, “A new microscopic principle,” Nature (London) 161, 777-778 (1948).

2. J. J. Barton, "Photoelectron Holography," Phys. Rev. Lett. 61, Num 12, 1356 (1988).

3. J. J. Barton, "Removing multiple Scattering and Twin Images from Holographic Images," Phys. Rev. Lett. 67, Num 22, 3106 (1991).

4. J. Sheng, E. Malkiel, and J. Katz, "Digital holographic microscope for measuring three-dimensional particle distributions and motions," Appl. Opt. 45, 3893-3901 (2006).

5. W. Xu, M. H. Jericho, I. A. Meinertzhagan and H. J. Kreuzer, “Digital in-line holography of microspheres,” Appl. Opt. 41, 5367 (2002).

6. L. Repetto, E. Piano and C. Pontiggia, "Lensless digital holographic microscope with light-emitting diode illumination," Opt. Lett. 29, 11321134 (2004).

7. J. P. Ryle, U. Gopinathan, S. McDonnell, T. J. Naughton, and J. T. Sheridan, "Digital in-line holography of biological specimens," Proc. SPIE 6311, 63110C (2006), DOI:10.1117/12.680798.

8. A. Khmaladze, M. Kim, and C.-M. Lo, "Phase imaging of cells by simultaneous dual-wavelength reflection digital holography," Opt. Express 16, 10900-10911 (2008).

9. W. Xu, M. J. Jericho, H. J. Kreuzer, and I. A. Meinertzhagen, "Tracking particles in four dimensions with in-line holographic microscopy," Opt. Lett. 28, 164-166 (2003).

10. L. Repetto, R. Chittofrati, E. Piano, and C. Pontiggia, "Infrared lensless holographic microscope with a vidicon camera for inspection of metallic evaporations on silicon wafers," Opt. Commun. 251, 44-50 (2005).

11. G. Pedrini and H. J. Tiziani, "Short-Coherence Digital Microscopy by Use of a Lensless Holographic Imaging System," Appl. Opt. 41, 4489-4496 (2002).

12. U. Gopinathan, G. Pedrini, and W. Osten, "Coherence effects in digital in-line holographic microscopy," J. Opt. Soc. Am. A 25, $2459-2466$ (2008).

13. J. Cheng and S. Han, "On X-ray in-line Gabor holography with a partially coherent source," Opt. Commun. 172, 17-24 (1999).

14. J. C. Dainty, "Laser speckle and related phenomena," (Springer, 1984).

15. K. M. Molony, J. P. Ryle, S. McDonnell, T. J. Naughton and J. T. Sheridan, "Segmentation and visualization of digital in-line holographic microscopy three-dimensional scenes using reconstructed intensity images," Proc. SPIE 7443, in progress

16. J. Garcia-Sucerquia, W. Xu, S. K. Jericho, P. Klages, M. H. Jericho, and H. J. Kreuzer, "Digital in-line holographic microscopy,” Appl. Opt. 45, pp. 836-850, 2006.

17. I. Yamaguchi and T. Zhang, "Phase-shifting digital holography," Opt. Lett. 22, 1268-1270 (1997).

18. Y. Zhang, G. Pedrini, W. Osten, and H. J. Tiziani, "Reconstruction of in-line digital holograms from two intensity measurements," Opt. Lett. 29, pp. 1287-1789, (2004).

19. G.Situ, J. P. Ryle, U. Gopinathan, and J. T. Sheridan, "Generalized in-line digital holographic technique based on intensity measurements at two different planes," Appl. Opt. 47, 711-717 (2008).

20. E. Leith and J. Upatnieks, "Reconstruced Wavefronts and Communication theory," J. Opt. Soc. Am. A. 52, 1123 (1962).

21. E. N. Leith and J. Upatnieks, "Wavefront reconstruction with diffused illumination and three-dimensional objects,” J. Opt. Soc. Am. 54, pp. 1295-1301, (1964).

22. B. M. Hennelly and J. T. Sheridan, "Optical encryption and the space bandwidth product," Opt. Commun. 247, 291-305 (2005).

23. ATCC website, "http://www.atcc.org/ATCCAdvancedCatalogSearch/ProductDetails/tabid/452/Default.aspx?ATCCNum=HTB22\&Template=cellBiology," (2009).

24. ATCC website, "http://www.atcc.org/ATCCAdvancedCatalogSearch/ProductDetails/tabid/452/Default.aspx?ATCCNum=HTB26\&Template=cellBiology," (2009).

25. M. Wurm, "Production of recombinant protein therapeutics in cultivated mammalian cells," Nature Biotechnology 22, 1393-1398 (2004).

26. D.C. Ghiglia \& L. A. Romero, "Robust two-dimensional weighted and unweighted phase unwrapping that uses fast transforms and iterative methods," J. Opt. Soc. Am. A 11, No. 1, 107 (1994).

27. M. A. Schofield \& Y. Zhu, "Fast Phase unwrapping algorithm for interferometric applications," Opt. Lett. 28, 1194 (2003).

28. U. Schnars and W. P. Jüptner, "Digital recording and numerical reconstruction of holograms,” Meas. Sci. Technol. 13, R85 (2002). 